\title{
Development and Plasticity of Cognitive Flexibility in Early and Middle Childhood
}

\author{
Frances Buttelmann ${ }^{1,2,3 *}$ and Julia Karbach ${ }^{1,2,4}$ \\ ${ }^{1}$ Department of Psychology, Goethe University Frankfurt, Frankfurt, Germany, ${ }^{2}$ Center for Research on Individual \\ Development and Adaptive Education of Children at Risk (IDeA), Frankfurt, Germany, ${ }^{3}$ Department of Developmental \\ Psychology, Friedrich Schiller University Jena, Jena, Germany, ${ }^{4}$ Department of Psychology, University of Koblenz-Landau, \\ Landau, Germany
}

\section{OPEN ACCESS}

Edited by:

Mike Wendt,

Medical School Hamburg, Germany

Reviewed by:

Claudia C. von Bastian,

Bournemouth University,

United Kingdom

Gregoire Borst,

Université Paris Descartes, France

*Correspondence:

Frances Buttelmann

frances.buttelmann@uni-jena.de

Specialty section:

This article was submitted to

Cognition,

a section of the journa

Frontiers in Psychology

Received: 31 January 2017

Accepted: 06 June 2017

Published: 20 June 2017

Citation:

Buttelmann F and Karbach J (2017)

Development and Plasticity

of Cognitive Flexibility in Early

and Middle Childhood.

Front. Psychol. 8:1040.

doi: 10.3389/fpsyg.2017.01040
Cognitive flexibility, the ability to flexibly switch between tasks, is a core dimension of executive functions (EFs) allowing to control actions and to adapt flexibly to changing environments. It supports the management of multiple tasks, the development of novel, adaptive behavior and is associated with various life outcomes. Cognitive flexibility develops rapidly in preschool and continuously increases well into adolescence, mirroring the growth of neural networks involving the prefrontal cortex. Over the past decade, there has been increasing interest in interventions designed to improve cognitive flexibility in children in order to support the many developmental outcomes associated with cognitive flexibility. This article provides a brief review of the development and plasticity of cognitive flexibility across early and middle childhood (i.e., from preschool to elementary school age). Focusing on interventions designed to improve cognitive flexibility in typically developing children, we report evidence for significant training and transfer effects while acknowledging that current findings on transfer are heterogeneous. Finally, we introduce metacognitive training as a promising new approach to promote cognitive flexibility and to support transfer of training.

Keywords: cognitive flexibility, intervention, childhood, executive functions, metacognition, DCCS, task switching

\section{INTRODUCTION}

Cognitive flexibility, the ability to shift between different tasks or goals, is considered a key aspect of executive functions (EF) allowing individuals to regulate their thoughts and actions adaptively (e.g., Miyake et al., 2000; Jurado and Rosselli, 2007). In the literature, it is also referred to by shifting, attention switching, or task switching, and includes both the ability to disengage from irrelevant information in a previous task and to focus on relevant information in a forthcoming task (Monsell, 2003). Thus, cognitive flexibility enables to think divergently, change perspective and adapt to a continuously changing environment.

When it comes to the structure of EF, earlier models have either assumed that it is a unitary construct (e.g., Duncan et al., 1997) or a set of dissociable control components (e.g., Stuss and Alexander, 2000). More recent approaches have shown the unity and diversity of EF in integrative frameworks (e.g., Miyake et al., 2000; Garon et al., 2008). The Miyake model, for instance, assumes that the core EF skills entail working memory (WM), inhibitory control, and cognitive flexibility. Importantly, this structure is subject to developmental changes, with a shift from a single latent EF 
factor to separate component processes from early childhood to school age and adolescence (e.g., Huizinga et al., 2006; Wiebe et al., 2008, 2011).

Importantly, EF in general and cognitive flexibility in particular contributes to a number of important life outcomes, such as academic achievement (review: Titz and Karbach, 2014). Colé et al. (2014), for instance, showed that cognitive flexibility predicted reading skills in second graders and a recent meta-analysis showed that cognitive flexibility was a significant predictor for both math and reading skills in children between the ages of 4 and 13 years (Yeniad et al., 2013). Given the strong relationship between flexibility and achievement, it is not surprising that many studies have aimed at training flexibility in order to improve children's performance in the classroom (review: Titz and Karbach, 2014; meta-analysis: Schwaighofer et al., 2015). We will focus on such training effects in the last section of this review. In the upcoming section, we will first describe the development of cognitive flexibility.

\section{DEVELOPMENT OF COGNITIVE FLEXIBILITY}

Infants within their first year of life already exhibit fundamental forms of EF (Carpenter et al., 1998), but the core components (WM, inhibition and flexibility; Miyake et al., 2000) rapidly develop during the preschool years (Hughes, 1998). Research focusing on the development across the lifespan demonstrates that EF continues developing throughout childhood (e.g., Davidson et al., 2006) well into adolescence (e.g., Huizinga and van der Molen, 2007) and early adulthood (e.g., Anderson et al., 2001). In this review, however, our focus will be on the preschool and elementary-school age. We will illustrate developmental changes in flexibility by referring to two widely used paradigms assessing children's cognitive flexibility, the Dimensional Change Card Sort task (DCCS; Zelazo, 2006) and the task-switching paradigm (Monsell, 2003).

Most studies investigating preschoolers applied the DCCS to test cognitive flexibility. In this task, children are shown cards with pictures displaying two dimensions (e.g., color and shape) and are told to sort these cards by one dimension (e.g., by color) (pre-switch phase). At some point, participants are told to sort the cards by the other dimension (i.e., by shape) (post-switch phase). While children from the age of 4 years are able to switch the rules, 3 -years-old typically perseverate and keep applying the first rule when they should apply the second one (e.g., Zelazo, 2006; Doebel and Zelazo, 2015). Performance continues to improve with age, as children are able to apply higher-order rules and handle more complex tasks (e.g., Chevalier and Blaye, 2009; Diamond, 2013), such as the task-switching paradigm. In this task, children are instructed to perform two tasks (A and B), e.g., two simple categorization tasks. In single-task blocks, participants perform both tasks separately (e.g., AAA, BBB), but in mixed-task blocks, they have to switch between both tasks (e.g., AABBAABB). This paradigm allows assessing two different components of cognitive flexibility - the ability to switch from one rule/task to another as well as the maintenance and selection of task sets in WM.
Karbach and Kray (2007) tested 5- to 6-years-old and 9-years-old on a cued task-switching paradigm. In task A, children had to categorize stimuli as either fruits or animals and in task B they had to indicate if the picture was presented in color or gray. Results showed an age-related improvement in the ability to maintain and select tasks, but not in the ability to switch between tasks. These different developmental trajectories of the processes subserving cognitive flexibility were confirmed by other studies applying switching tasks and investigating a wider range of ages (e.g., Cepeda et al., 2001; Crone et al., 2004; Reimers and Maylor, 2005; Huizinga and van der Molen, 2007; Kray et al., 2008). For instance, Huizinga and van der Molen (2007) examined the developmental change in switching and maintenance and found that children reached adult levels of switching abilities by the age of 11 years, while task maintenance abilities only matured at the age of 15 years. In sum, these findings point to an earlier maturation of task-switching than task-maintenance and selection abilities.

Developmental trajectories of $\mathrm{EF}$ are have been linked to maturational changes of the prefrontal cortex (PFC) and associated cortical and subcortical structures, including parietal regions and basal ganglia (e.g., Casey et al., 2005; Bunge and Wright, 2007). Some regions within the PFC, such as the orbitofrontal cortex, reach structural maturity at an earlier age, whereas others, such as the dorsolateral PFC, show a more protracted maturational time course (Gogtay et al., 2004). There is evidence - including studies using the DCCS and the task-switching paradigm - suggesting that those differences in structural maturation are paralleled by changes in functional maturation and hence may account for distinct developmental trajectories among EFs (Bunge and Zelazo, 2006).

For instance, a study by Moriguchi and Hiraki (2009) assessed 3- and 5-year-old children as well as adults with the DCCS task using NIRS (near-infrared spectroscopy). Results for the 3 -years-old indicated that only some 3-years-old who passed the task showed significant activation in the right inferior PFC. In contrast, 5-years-old and adults showed this activation bilaterally (see also Moriguchi and Hiraki, 2014). This finding was consistent with another longitudinal study (Moriguchi and Hiraki, 2011) testing children at the age of 3 and 4 years. In contrast to age 3 , children at age 4 passed the task and showed an increasing activation in the left inferior PFC (cf. Morton et al., 2009). Together with the finding that functional connectivity between the lateral PFC and inferior parietal cortex increases as children age (Ezekiel et al., 2013), these findings add to the evidence indicating that the PFC is a key player in the development of cognitive flexibility.

Studies using a task-switching paradigm confirm these age differences in brain activation. Rubia et al. (2006), for instance, found age-related increases in the recruitment of several brain regions that have been implicated in cognitive flexibility, including right inferior PFC, left parietal cortex, anterior cingulate cortex (ACC), and striatum. Moreover, there is neuroscientific evidence supporting the different developmental trajectories of task switching and task maintenance/selection: Crone et al. (2006) tested children, adolescents and adults and found an adult-like pattern of activation for task switching in the 
pre-supplementary motor area by adolescence. In contrast, the activation for task maintenance and selection in the ventrolateral PFC differed among children, adolescents, and adults (see Wendelken et al., 2012, for similar patterns of activation in children and adults, but different timing, pointing more to a change in the temporal dynamics rather than qualitative changes during development).

Taken together, the behavioral and neuroimaging results demonstrate that cognitive flexibility rapidly increases during early and middle childhood, suggesting that this may be a period of high plasticity and malleability sensitive to developmental as well as environmentally driven changes. It is not surprising then that much research focused on interventions designed to support the development of EF. These interventions range from school and curriculum-based programs to physical and cognitive training regimes (for reviews see Diamond, 2012; Karbach and Unger, 2014).

\section{PLASTICITY OF COGNITIVE FLEXIBILITY - TRAINING AND TRANSFER EFFECTS}

When it comes to training of EF, most of the existing developmental studies have certainly targeted WM (for reviews see Könen et al., 2016; Rueda et al., 2016). However, there are a handful of studies training cognitive flexibility in early and middle childhood. While some have trained multiple components of EF at the same time (e.g., Röthlisberger et al., 2012; Traverso et al., 2015), others have focused specifically on cognitive flexibility. We will illustrate this line of research by reviewing interventions applying the DCCS and the task-switching paradigm. We will report training effects and also evidence for transfer of training-related gains to untrained tasks and abilities, which recently has been discussed very controversially in the community (e.g., Shipstead et al., 2012).

Kloo and Perner (2003) trained 3- and 4-year-old children on the DCCS. Before and after training, the children performed the DCCS and a false-belief task (as well as a number of control tasks) including a novel version of the DCCS with different test and target cards at post-test. Children in the DCCS training group showed larger improvements on the DCCS and the false-belief task than children in the control group. They also outperformed the control group on the novel DCCS task. Thus, training did not only benefit cognitive flexibility but also transferred to false-belief understanding. Also training DCCS performance, van Bers et al. (2014) studied the effects of feedback on cognitive flexibility in 3-years-old. Providing feedback on the post-switch sorting improved DCCS performance compared to a standard condition without feedback. Importantly, these gains transferred to a novel version of the DCCS administered immediately after training as well as 1 week later.

In school-aged children, a number of studies have applied the task-switching paradigm to train cognitive flexibility. Adopting a lifespan approach, Cepeda et al. (2001) tested a sample ranging from 7-82 years of age on single-task and mixed-task blocks $(N=152)$. After three sessions of training, participants - and particularly children - significantly improved task maintenance and selection (Kray et al., 2008).

Following up on these training gains, other studies investigated whether task-switching training also transfers to untrained tasks and domains (e.g., Karbach and Kray, 2009; Zinke et al., 2012). Karbach and Kray (2009) had children (8-10 years of age) as well as younger and older adults $(N=168)$ perform four sessions of task-switching training. Results showed that training improved performance in an untrained switching task compared to a control group performing single-task training. Further, training also improved inhibition, verbal and visuo-spatial WM and fluid intelligence. Based on the transfer to WM and inhibition, another study tested the effects of task-switching training in children with ADHD because they usually show significant deficits in these domains. And indeed, four sessions of switching training resulted in significant improvements in an untrained switching task, inhibition and WM in 7- to 12-year-old boys with $\mathrm{ADHD}(N=20$; Kray et al., 2012).

These findings indicate that training cognitive flexibility may be a key factor for improving other dimensions of EF. Still, it has to be noted that transfer was less pronounced in other studies: Zinke et al. (2012) assessed the effects of taskswitching training in 10 - to 14 -years-old $(N=80)$. After three sessions of training, participants showed significant training gains and also transfer to an untrained switching task, but no transfer to inhibition. These effects mirror data from 8- to 11-years-old performing task-switching training embedded in a game environment (Dörrenbächer et al., 2014).

Thus, training regimes based on the DCCS and task-switching yielded significant improvements in cognitive flexibility across childhood and adolescence. Moreover, there is evidence showing that they can result in transfer to other EF dimensions, even though results on transfer of switching training are heterogeneous, just as they are for other types of cognitive training (for reviews, see Karbach and Kray, 2016; Könen et al., 2016). However, the existing studies almost exclusively analyzed data on the group level and ignored individual differences in training-induced gains. Given that even individuals participating in exactly the same training regime usually highly differ in their training outcomes (for reviews see Könen and Karbach, 2015; Katz et al., 2016), it is crucial to study individual differences in baseline performance as well as the individual performance development during training to understand these differential outcomes. Previous studies, for instance, showed that EF training often resulted in compensation effects, indicating that participants with lower baseline performances benefitted more (e.g., Cepeda et al., 2001; Bherer et al., 2008; Karbach and Kray, 2009; Zinke et al., 2012) and that individual differences in age and fluid intelligence (Bürki et al., 2014), motivational aspects (Katz et al., 2016), and the amount of training gain (e.g., Jaeggi et al., 2011) contributed to the success of training interventions. However, the underlying mechanisms are still largely unknown, especially in early childhood.

Another aspect that gains more and more attention in the field of training research is the question which aspects of intervention designs moderate training-induced gains. 
While current meta-analyses have tested effects related to the intensity, frequency and adaptivity of training, just to name a few (e.g., Karbach and Verhaeghen, 2014; Au et al., 2015; Schwaighofer et al., 2015), other features - such as the instructional design of training - have received less attention. However, since EF entails higher-level cognitive processes, it has been proposed that metacognitive processes, i.e., reflecting on one's own thinking and actions, may be important for the development and plasticity of EF (e.g., Zelazo et al., 2003; Chevalier and Blaye, 2016). This aspect has been investigated in a few recent studies. Espinet et al. (2013) showed across three experiments that training with corrective feedback and instruction to reflect on the task led to substantial improvements in DCCS performance in 2- to 4-years-old. Compared to controls, trained children benefitted more on an untrained version of the DCCS. Moreover, they showed a significant reduction of the $\mathrm{N} 2$ amplitude (an indicator of conflict detection) during DCCS performance and at the same time an increase in reaction time. The authors concluded that slowing down may have provided the time needed to reflect on the hierarchical nature of the DCCS task and to resolve the conflict inherent in the task (Espinet et al., 2012).

Similarly, Moriguchi et al. (2015) trained 3- to 5-year-old preschoolers on the DCCS in two experiments. Children performed a pre-test, training and a post-test. In the experimental group, they interacted with a puppet and were asked to explain the task with all the rules to the puppet, to think about task demands or possible strategies to solve the task in order to foster metacognitive reflection. Results showed that the experimental group improved from pre-test to post-test and performed significantly better than the control group at post-test. Moreover, using NIRS Moriguchi et al. (2015) showed a higher activation in the left PFC after training, again confirming the importance of the PFC for EF.

There is also evidence from task switching: Chevalier and Blaye (2016) investigated whether children's EF monitoring drives EF development from 6 to 10 years of age. They recorded gaze position while participants performed a self-paced task-switching paradigm. In this task, the children had as much time as they needed to proactively prepare for the next task. Both the analysis of gaze trajectories and performance showed that older children were better prepared than younger ones when they responded, even though younger participants could have taken more time to prepare their response. Thus, with increasing age children are better able to monitor EF engagement, pointing to the important contribution of metacognitive processes to $\mathrm{EF}$ development.

Even though these findings highlight the importance of metacognition for efficient EF functioning, metacognitive instructions have rarely been applied in cognitive-training research. Unlike many previous training approaches, metacognitive EF training would not aim to enhance the quantity of EF that children can engage, but to change qualitatively how they engage EF as a function of task difficulty (for an example of metacognitive training in reasoning research see
Houdé et al., 2000, 2001). Thus, metacognitive training should facilitate the flexible adaptation to new tasks by training the children to reflect on how to approach them, for instance integrating information about current task demands and past experiences in order to weigh the respective costs (e.g., mental effort) and benefits (e.g., rewards) of available control strategies (cf. Chevalier and Blaye, 2016). Metacognitive training should further encourage performance evaluation, including error detection and feedback processing, all of which are still gradually developing in young children (e.g., Chevalier et al., 2009; Andersen et al., 2014; DuPuis et al., 2014). Given that this metacognitive approach is relatively task-unspecific, it may even support transfer of flexibility training to untrained tasks and abilities. Future studies may want to consider this promising approach when designing new interventions to improve cognitive flexibility (or EF in general).

\section{CONCLUSION}

Cognitive flexibility develops rapidly during the preschool years and continues to improve across adolescence and young adulthood. Given that EF, and cognitive flexibility in particular, are related to many important life outcomes including academic achievement (e.g., mathematics or reading skills; Yeniad et al., 2013; Titz and Karbach, 2014) and even health status during adulthood (Moffitt et al., 2011), numerous interventions have been designed to improve childhood EF.

Recent training studies provided accumulating evidence for the trainability of cognitive flexibility in early and middle childhood. We illustrated these training effects and also findings on transfer based on studies applying the DCCS and the task-switching paradigm. Training on both tasks has been shown to transfer to other dimensions of EF and to core dimensions of theory of mind, such as false-belief understanding. Importantly, these effects were not only present on the behavioral level but also mirrored by eye-tracking and neuroscientific measures. Given that the mechanisms underlying these training and transfer effects are not fully understood, future studies should try disentangling them, possibly by considering individual differences in training outcomes and by testing the role of metacognitive processes in the plasticity of cognitive flexibility in childhood.

\section{AUTHOR CONTRIBUTIONS}

All authors listed have made a substantial, direct and intellectual contribution to the work, and approved it for publication.

\section{FUNDING}

This research was funded by a grant from the German Research Foundation (DFG) awarded to JK (KA 3216/2-1). 


\section{REFERENCES}

Andersen, L. M., Visser, I., Crone, E. A., Koolschijn, P. C., and Raijmakers, M. E. (2014). Cognitive strategy use as an index of developmental differences in neural responses to feedback. Dev. Psychol. 50, 2686-2696. doi: 10.1037/a0038106

Anderson, V. A., Anderson, P., Northam, E., Jacobs, R., and Catroppa, C. (2001). Development of executive functions through late childhood and adolescence in an Australian sample. Dev. Neuropsychol. 20, 385-406. doi: 10.1207/ S15326942DN2001_5

Au, J., Sheehan, E., Tsai, N., Duncan, G. J., Buschkuehl, M., and Jaeggi, S. M. (2015). Improving fluid intelligence with training on working memory: a meta-analysis. Psychon. Bull. Rev. 22, 366-377. doi: 10.3758/s13423-014-0699-x

Bherer, L., Kramer, A. F., Peterson, M. S., Colcombe, S., Erickson, K., and Becic, E. (2008). Transfer effects in task-set cost and dual-task cost after dual-task training in older and younger adults: further evidence for cognitive plasticity in attentional control in late adulthood. Exp. Aging Res. 34, 188-219. doi: 10.1080/03610730802070068

Bunge, S. A., and Wright, S. B. (2007). Neurodevelopmental changes in working memory and cognitive control. Curr. Opin. Neurobiol. 17, 243-250. doi: 10.1016/j.conb.2007.02.005

Bunge, S. A., and Zelazo, P. D. (2006). A brain-based account of the development of rule use in childhood. Curr. Dir. Psychol. Sci. 15, 118-121.

Bürki, C. N., Ludwig, C., Chicherio, C., and de Ribaupierre, A. (2014). Individual differences in cognitive plasticity: an investigation of training curves in younger and older adults. Psychol. Res. 78, 821-835. doi: 10.1007/s00426-014-0559-3

Carpenter, M., Nagell, K., Tomasello, M., Butterworth, G., and Moore, C. (1998). Social cognition, joint attention, and communicative competence from 9 to 15 months of age. Monogr. Soc. Res. Child Dev. 63, 1-143. doi: 10.2307/1166214

Casey, B. J., Tottenham, N., Liston, C., and Durston, S. (2005). Imaging the developing brain: what have we learned about cognitive development? Trends Cogn. Sci. 9, 104-110. doi: 10.1016/j.tics.2005.01.011

Cepeda, N. J., Kramer, A. F., and Gonzalez de Sather, J. C. M. (2001). Changes in executive control across the life span: examination of task-switching performance. Dev. Psychol. 37, 715-730. doi: 10.1037//0012-1649.37.5.715

Chevalier, N., and Blaye, A. (2009). Setting goals to switch between tasks: effect of cue transparency on children's cognitive flexibility. Dev. Psychol. 45, 782-797. doi: $10.1037 / \mathrm{a} 0015409$

Chevalier, N., and Blaye, A. (2016). Metacognitive monitoring of executive control engagement during childhood. Child Dev. 87, 1264-1276. doi: 10.1111/cdev. 12537

Chevalier, N., Dauvier, B., and Blaye, A. (2009). Preschoolers' use of feedback for flexible behavior: insights from a computational model. J. Exp. Child Psychol. 103, 251-267. doi: 10.1016/j.jecp.2009.03.002

Colé, P., Duncan, L. G., and Blaye, A. (2014). Cognitive flexibility predicts early reading skills. Front. Psychol. 5:565. doi: 10.3389/fpsyg.2014.00565

Crone, E. A., Donohue, S. E., Honomichl, R., Wendelken, C., and Bunge, S. A. (2006). Brain regions mediating flexible rule use during development. J. Neurosci. 26, 11239-11247. doi: 10.1523/jneurosci.2165-06.2006

Crone, E. A., Ridderinkhof, K. R., Worm, M., Somsen, R. J. M., and van der Molen, M. W. (2004). Switching between spatial stimulus-response mappings: a developmental study of cognitive flexibility. Dev. Sci. 7, 443-455. doi: 10.1111/ j.1467-7687.2004.00365.x

Davidson, M. C., Amso, D., Anderson, L. C., and Diamond, A. (2006). Development of cognitive control and executive functions from 4 to 13 years: evidence from manipulations of memory, inhibition, and task switching. Neuropsychologia 44, 2037-2078. doi: 10.1016/j.neuropsychologia.2006.02.006

Diamond, A. (2012). Activities and programs that improve children's executive functions. Curr. Dir. Psychol. Sci. 21, 335-341. doi: 10.1177/09637214124 53722

Diamond, A. (2013). Executive functions. Annu. Rev. Psychol. 64, 135-168. doi: 10.1146/annurev-psych-113011-143750

Doebel, S., and Zelazo, P. D. (2015). A meta-analysis of the Dimensional Change Card Sort: implications for developmental theories and the measurement of executive function in children. Dev. Rev. 38, 241-268. doi: 10.1016/j.dr.2015. 09.001

Dörrenbächer, S., Müller, P. M., Tröger, J., and Kray, J. (2014). Dissociable effects of game elements on motivation and cognition in a task-switching training in middle childhood. Front. Psychol. 5:1275. doi: 10.3389/fpsyg.2014.01275
Duncan, J., Johnson, R., Swales, M., and Freer, C. (1997). Frontal lobe deficits after head injury: unity and diversity of function. Cogn. Neuropsychol. 14, 713-741. doi: 10.1080/026432997381420

DuPuis, D., Ram, N., Willner, C. J., Karalunas, S., Segalowitz, S. J., and GatzkeKopp, L. M. (2014). Implications of ongoing neural development for the measurement of the error-related negativity in childhood. Dev. Sci. 18, 452-468. doi: $10.1111 /$ desc. 12229

Espinet, S. D., Anderson, J. E., and Zelazo, P. D. (2012). N2 amplitude as a neural marker of executive function in young children: an ERP study of children who switch versus perseverate on the Dimensional Change Card Sort. Dev. Cogn. Neurosci. 2, 49-58. doi: 10.1016/j.dcn.2011.12.002

Espinet, S. D., Anderson, J. E., and Zelazo, P. D. (2013). Reflection training improves executive function in preschool-age children: behavioral and neural effects. Dev. Cogn. Neurosci. 4, 3-15. doi: 10.1016/j.dcn.2012.11.009

Ezekiel, F., Bosma, R., and Morton, J. B. (2013). Dimensional change card sort performance associated with age-related differences in functional connectivity of lateral prefrontal cortex. Dev. Cogn. Neurosci. 5, 40-50. doi: 10.1016/j.dcn. 2012.12.001

Garon, N., Bryson, S. E., and Smith, I. M. (2008). Executive function in preschoolers: a review using an integrative framework. Psychol. Bull. 134, 31-60. doi: 10.1037/0033-2909.134.1.31

Gogtay, N., Giedd, J. N., Hayashi, K. M., Greenstein, D., Vaituzis, C., Nugent, T. F., et al. (2004). Dynamic mapping of human cortical development during childhood through early adulthood. Proc. Natl. Acad. Sci. U.S.A. 101, 8174-8179. doi: 10.1073/pnas.0402680101

Houdé, O., Zago, L., Crivello, F., Moutier, S., Pineau, A., Mazoyer, B., et al. (2001). Access to deductive logic depends on a right ventromedial prefrontal area devoted to emotion and feeling. Neuroimage 14, 1486-1492. doi: 10.1006/nimg. 2001.0930

Houdé, O., Zago, L., Mellet, E., Moutier, S., Pineau, A., Mazoyer, B., et al. (2000). Shifting from the perceptual brain to the logical brain. J. Cogn. Neurosci. 12, 721-728. doi: 10.1162/089892900562525

Hughes, C. (1998). Executive function in preschoolers: links with theory of mind and verbal ability. Br. J. Dev. Psychol. 16, 233-253. doi: 10.1111/j.2044-835X. 1998.tb00921.x

Huizinga, M., Dolan, C. V., and van der Molen, M. W. (2006). Age-related change in executive function: developmental trends and a latent variable analysis. Neuropsychologia 44, 2017-2036. doi: 10.1016/j.neuropsychologia.2006.01.010

Huizinga, M., and van der Molen, M. W. (2007). Age-group differences in setswitching and set-maintenance on the Wisconsin Card Sorting Task. Dev. Neuropsychol. 31, 193-215. doi: 10.1080/87565640701190817

Jaeggi, S. M., Buschkuehl, M., Jonides, J., and Shah, P. (2011). Short-and long-term benefits of cognitive training. Proc. Natl. Acad. Sci. U.S.A. 108, 10081-10086. doi: $10.1073 /$ pnas. 1103228108

Jurado, M. B., and Rosselli, M. (2007). The elusive nature of executive functions: a review of our current understanding. Neuropsychol. Rev. 17, 213-233. doi: $10.1007 / \mathrm{s} 11065-007-9040-\mathrm{z}$

Karbach, J., and Kray, J. (2007). Developmental changes in switching between mental task sets: the influence of verbal labeling in childhood. J. Cogn. Dev. 8, 205-236. doi: 10.1080/15248370701202430

Karbach, J., and Kray, J. (2009). How useful is executive control training? Age differences in near and far transfer of task-switching training. Dev. Sci. 12, 978-990. doi: 10.1111/j.1467-7687.2009.00846.x

Karbach, J., and Kray, J. (2016). "Executive functions," in Cognitive Training - An Overview of Features and Applications, eds T. Strobach and J. Karbach (Cham: Springer International), 93-103.

Karbach, J., and Unger, K. (2014). Executive control training from middle childhood to adolescence. Front. Psychol. 5:390. doi: 10.3389/fpsyg.2014.00390

Karbach, J., and Verhaeghen, P. (2014). Making working memory work a metaanalysis of executive-control and working memory training in older adults. Psychol. Sci. 25, 2027-2037. doi: 10.1177/0956797614548725

Katz, B., Jones, M. R., Shah, P., Buschkühl, M., and Jaeggi, S. M. (2016). "Individual differences and motivational effects," in Cognitive Training - An Overview of Features and Applications, eds T. Strobach and J. Karbach (Cham: Springer International), 157-166.

Kloo, D., and Perner, J. (2003). Training transfer between card sorting and false belief understanding: helping children apply conflicting descriptions. Child Dev. 74, 1823-1839. doi: 10.1046/j.1467-8624.2003.00640.x 
Könen, T., and Karbach, J. (2015). The benefits of looking at intraindividual dynamics in cognitive training data. Front. Psychol. 6:615. doi: 10.3389/fpsyg. 2015.00615

Könen, T., Strobach, T., and Karbach, J. (2016). "Working memory," in Cognitive Training - An Overview of Features and Applications, eds T. Strobach and J. Karbach (Cham: Springer International), 59-68.

Kray, J., Eber, J., and Karbach, J. (2008). Verbal self-instructions in task switching: a compensatory tool for action-control deficits in childhood and old age? Dev. Sci. 11, 223-236. doi: 10.1111/j.1467-7687.2008.00673.x

Kray, J., Karbach, J., Haenig, S., and Freitag, C. (2012). Can task-switching training enhance executive control functioning in children with attention deficit/hyperactivity disorder? Front. Hum. Neurosci. 5:180. doi: 10.3389/fnhum.2011. 00180

Miyake, A., Friedman, N. P., Emerson, M. J., Witzki, A. H., Howerter, A., and Wager, T. (2000). The unity and diversity of executive functions and their contributions to complex "frontal lobe" tasks: a latent variable analysis. Cogn. Psychol. 41, 49-100. doi: 10.1006/cogp.1999.0734

Moffitt, T. E., Arseneault, L., Belsky, D., Dickson, N., Hancox, R. J., Harrington, H., et al. (2011). A gradient of childhood self-control predicts health, wealth, and public safety. Proc. Natl. Acad. Sci. U.S.A. 108, 2693-2698. doi: 10.1073/pnas. 1010076108

Monsell, S. (2003). Task switching. Trends Cogn. Sci. 7, 134-140. doi: 10.1016/ S1364-6613(03)00028-7

Moriguchi, Y., and Hiraki, K. (2009). Neural origin of cognitive shifting in young children. Proc. Natl. Acad. Sci. U.S.A. 106, 6017-6021. doi: 10.1073/pnas. 0809747106

Moriguchi, Y., and Hiraki, K. (2011). Longitudinal development of prefrontal function during early childhood. Dev. Cogn. Neurosci. 1, 153-162. doi: 10.1016/ j.dcn.2010.12.004

Moriguchi, Y., and Hiraki, K. (2014). Neural basis of learning from television in young children. Trends Neurosci. Educ. 3, 122-127. doi: 10.1016/j.tine.2014. 07.001

Moriguchi, Y., Sakata, Y., Ishibashi, M., and Ishikawa, Y. (2015). Teaching others rule-use improves executive function and prefrontal activations in young children. Front. Psychol. 6:894. doi: 10.3389/fpsyg.2015.00894

Morton, J. B., Bosma, R., and Ansari, D. (2009). Age-related changes in brain activation associated with dimensional shifts of attention: an fMRI study. Neuroimage 46, 249-256. doi: 10.1016/j.neuroimage.2009.01.037

Reimers, S., and Maylor, E. A. (2005). Task switching across the life span: effects of age on general and specific switch costs. Dev. Psychol. 41, 661-671. doi: 10.1037/0012-1649.41.4.661

Röthlisberger, M., Neuenschwander, R., Cimeli, P., Michel, E., and Roebers, C. M. (2012). Improving executive functions in 5- and 6-year-olds: evaluation of a small group intervention in prekindergarten and kindergarten children. Infant Child Dev. 21, 411-429. doi: 10.1002/icd.752

Rubia, K., Smith, A. B., Woolley, J., Nosarti, C., Heyman, I., Taylor, E., et al. (2006). Progressive increase of frontostriatal brain activation from childhood to adulthood during event-related tasks of cognitive control. Hum. Brain Mapp. 27, 973-993. doi: 10.1002/hbm.20237

Rueda, M. R., Cómbita, L. M., and Pozuelos, J. P. (2016). "Childhood and adolescence," in Cognitive Training - An Overview of Features and
Applications, eds T. Strobach and J. Karbach (Cham: Springer International), 33-44.

Schwaighofer, M., Fischer, F., and Bühner, M. (2015). Does working memory training transfer? A meta-analysis including training conditions as moderators. Educ. Psychol. 50, 138-166. doi: 10.1080/00461520.2015.1036274

Shipstead, Z., Redick, T. S., and Engle, R. W. (2012). Is working memory training effective? Psychol. Bull. 138, 628-654. doi: 10.1037/a0027473

Stuss, D. T., and Alexander, M. P. (2000). Executive functions and the frontal lobes: a conceptual view. Psychol. Res. 63, 289-298.

Titz, C., and Karbach, J. (2014). Working memory and executive functions: effects of training on academic achievement. Psychol. Res. 78, 852-868. doi: 10.1007/ s00426-013-0537-1

Traverso, L., Viterbori, P., and Usai, M. C. (2015). Improving executive function in childhood: evaluation of a training intervention for 5-year-old children. Front. Psychol. 6:525. doi: 10.3389/fpsyg.2015.00525

van Bers, B. M., Visser, I., and Raijmakers, M. (2014). Preschoolers learn to switch with causally related feedback. J. Exp. Child Psychol. 126, 91-102. doi: 10.1016/ j.jecp.2014.03.007

Wendelken, C., Munakata, Y., Baym, C., Souza, M., and Bunge, S. A. (2012). Flexible rule use: common neural substrates in children and adults. Dev. Cogn. Neurosci. 2, 329-339. doi: 10.1016/j.dcn.2012.02.001

Wiebe, S. A., Espy, K. A., and Charak, D. (2008). Using confirmatory factor analysis to understand executive control in preschool children: I. Latent structure. Dev. Psychol. 44, 575-587. doi: 10.1037/0012-1649.44.2.575

Wiebe, S. A., Sheffield, T., Nelson, J. M., Clark, C. A. C., Chevalier, N., and Espy, K. A. (2011). The structure of executive function in 3-year-olds. J. Exp. Child Psychol. 108, 436-452. doi: 10.1016/j.jecp.2010.08.008

Yeniad, N., Malda, M., Mesman, J., van Ijzendoorn, M. H., and Pieper, S. (2013). Shifting ability predicts math and reading performance in children: a metaanalytical study. Learn. Individ. Differ. 23, 1-9. doi: 10.1016/j.lindif.2012. 10.004

Zelazo, P. D. (2006). The Dimensional Change Card Sort: a method of assessing executive function in children. Nat. Protoc. 1, 297-301. doi: 10.1038/nprot. 2006.46

Zelazo, P. D., Muller, U., Frye, D., and Marcovitch, S. (2003). The development of executive function. Monogr. Soc. Res. Child Dev. 68, vii-137. doi: 10.1111/j. 0037-976x.2003.00261.x

Zinke, K., Einert, M., Pfennig, L., and Kliegel, M. (2012). Plasticity of executive control through task switching training in adolescents. Front. Hum. Neurosci. 6:41. doi: 10.3389/fnhum.2012.00041

Conflict of Interest Statement: The authors declare that the research was conducted in the absence of any commercial or financial relationships that could be construed as a potential conflict of interest.

Copyright (c) 2017 Buttelmann and Karbach. This is an open-access article distributed under the terms of the Creative Commons Attribution License (CC BY). The use, distribution or reproduction in other forums is permitted, provided the original author(s) or licensor are credited and that the original publication in this journal is cited, in accordance with accepted academic practice. No use, distribution or reproduction is permitted which does not comply with these terms. 